\title{
In vitro Activity of Meropenem-Vaborbactam versus Other Antibiotics Against Carbapenem-Resistant Escherichia coli from Southeastern China
}

\author{
Na Huang' \\ Tao Chen' \\ Liqiong Chen ${ }^{2}$ \\ Ying Zhang ${ }^{2}$ \\ Yishuai $\operatorname{Lin}^{2}$ \\ Xiangkuo Zheng' \\ Tieli Zhou' \\ Lijiang Chen' \\ 'Department of Clinical Laboratory, The \\ First Affiliated Hospital of Wenzhou \\ Medical University, Wenzhou, 325035 , \\ Zhejiang Province, People's Republic of \\ China; ${ }^{2}$ Department of Medical Lab \\ Science, School of Laboratory Medicine \\ and Life Science, Wenzhou Medical \\ University, Wenzhou, 325035, Zhejiang \\ Province, People's Republic of China
}

Correspondence: Lijiang Chen; Tieli Zhou Department of Clinical Laboratory, The First Affiliated Hospital of Wenzhou Medical University, Wenzhou, Zhejiang Province, People's Republic of China Tel +86-577-8669-9370;

+86-577-86689885

Email wyychenlijiang@।63.com;

wyztli@I63.com
Purpose: This study aimed to evaluate the in vitro activity of meropenem-vaborbactam (MVB) against a collection of carbapenem-resistant Escherichia coli (CREC) isolates and to compare the activity with other antibiotics with regard to different separation sites, carbapenem-resistant mechanisms, and sequence types (STs).

Methods: A total of 58 CREC strains were used as the experimental strains from the First Affiliated Hospital of Wenzhou Medical University in southeastern China. The minimum inhibitory concentrations of MVB, ceftazidime-avibactam, and tigecycline against all the experimental strains were determined by the microdilution broth method.

Results: MVB exhibited higher antimicrobial activity ( $83 \%$ susceptibility) than that of other antibiotics, except for colistin and tigecycline. The susceptibility of CREC strains towards MVB varied with regard to carbapenem-resistant mechanisms and STs, especially in Klebsiella pneumoniae carbapenemase (KPC)-positive isolates and ST8 isolates.

Conclusion: MVB exhibited considerably high activity against KPC-producing and ST8 CREC isolates. It has the great potential to be an alternative for the treatment of infections caused by CREC after determining the type of carbapenemase, the susceptibility to MVB and/or STs.

Keywords: meropenem-vaborbactam, carbapenem-resistant Escherichia coli, in vitro activity, KPC-producing, ST8

\section{Introduction}

The emergence and spread of carbapenem-resistant Enterobacterales (CRE) are serious threats, ${ }^{1,2}$ leading to poor clinical outcomes of conventional antibiotic therapy, and especially carbapenem-resistant Escherichia coli (CREC), as one class of the most clinically relevant $\mathrm{CRE}$, has become a major threat in hospitals worldwide. Resistance in CREC is mainly attributed to the presence of carbapenemase encoded by plasmid, which should be closely monitored because of their potential trend to spread in both hospital and community settings. ${ }^{3,4}$ The currently available antibiotic options against CREC infections are limited to polymyxins, tigecycline, fosfomycin, and aminoglycosides as the mainstays. ${ }^{5,6}$ Nowadays, in clinic context, the CREC infections have raised serious concerns that common infections with these "superbugs" may soon be untreatable, which is necessary to exploit new and effective anti-CREC therapies.

Meropenem-vaborbactam (MVB), a new antibacterial drug, based on an old betalactam molecule with a novel beta/Betalactam inhibitor active on 
carbapenemases, was approved in August 2017 by the US Food and Drug Administration (FDA) for the treatment of complicated urine infections caused by Enterobacterales. ${ }^{7}$ MVB can slow down the development of bacterial resistance, and hence, is advantageous than meropenem. ${ }^{6,8}$ A study reported that MVB exhibits considerable activity against class $\mathrm{A}$ and $\mathrm{C}$ serine beta/Betalactams, including Klebsiella pneumoniae carbapenemase (KPC), imipenemhydrolyzing beta/Betalactam carbapenemase, Serratia marcescens enzymes, non-Metallo-carbapenemase-A, and Brazilian Klebsiella carbapenemase- $1 .{ }^{9}$ In addition, MVB monotherapy showed significant improvement in clinical cure rates, lower nephrotoxicity, and lower mortality rates. $^{7,8}$

In recent years, different resistance patterns against colistin and fluoroquinolone have been observed in E. coli isolates of different sequence types (STs). ${ }^{10-12}$ This study aimed to evaluate the in vitro activity of MVB against CREC isolates and to compare the activity with other antibiotics with regard to different separation sites, carbapenem-resistant mechanisms, and STs. This study provides important insights for the development of new effective therapeutic strategies against CREC infections.

\section{Materials and Methods}

\section{Bacterial Isolates and Resistance Mechanisms}

A total of 58 CREC isolates were isolated during our previous study ${ }^{13}$ from different clinical samples collected from the First Affiliated Hospital of Wenzhou Medical University, Wenzhou, China. These isolates were categorized into 19 different STs, among which the predominant was ST8. The isolates were isolated from different clinical samples, and most of them were isolated from urine samples. Various carbapenem-resistant mechanisms were identified mainly through polymerase chain reaction (PCR) and sequencing in our previous study, ${ }^{13}$ and $b l a_{\mathrm{NDM}}$ was the principal carbapenem-resistant mechanism.

\section{Antimicrobial Susceptibility Testing}

Meropenem, vaborbactam, ceftazidime, avibactam, and tigecycline were provided by The Medicines Company (MedChemExpress, USA). The minimum inhibitory concentrations (MICs) of MVB (the fixed concentration of vaborbactam: $8 \mu \mathrm{g} / \mathrm{mL}),{ }^{14}$ ceftazidime-avibactam (CAZ/ $\mathrm{AVI}$; the fixed concentration of avibactam: $4 \mu \mathrm{g} / \mathrm{mL}$ ), and tigecycline were evaluated by the microdilution broth method. The results were interpreted on the basis of the latest guidelines published by the Clinical and Laboratory Standards Institute (CLSI 2019; Pittsburgh, PA, USA). Quality control testing was performed by using E. coli ATCC 25922, and the quality control results were within the specified CLSI limits. All the experiments were performed in triplicate.

\section{Statistical Analysis}

Statistical analyses were performed using the SPSS 22.0 software (IBM, Armonk, NY, USA). The categorical variables were defined as the number and percentage of subjects. The Chi-square test or Fisher's exact test was performed to evaluate the differences in the categorical variables. A $p$-value of $<0.05$ was considered statistically significant.

\section{Results}

\section{Antimicrobial Susceptibility Testing of CREC for Commonly Used}

\section{Antimicrobials}

The $\mathrm{MIC}_{50}$ and $\mathrm{MIC}_{90}$ values and resistance rates of commonly used drugs against CREC are shown in Table 1 and our previous study. ${ }^{13}$ All 58 CREC strains exhibited lower susceptibility towards ampicillin (2\%), ceftriaxone $(2 \%)$, ceftazidime $(7 \%)$, ertapenem $(0)$, ciprofloxacin (0), levofloxacin (0), and gentamicin (14\%), compared with the susceptibility towards amikacin (78\%), fosfomycin (62\%) and imipenem (65\%). While colistin, tigecycline, CAZ/AVI and MVB were the last-line of defense drugs for the treatment CREC infection, with susceptibility rates of $0,100 \%, 60 \%$, and $83 \%$, respectively.

\section{MICs of MVB versus MICs of Meropenem}

Only 6 CREC strains $(6 / 58,10.34 \%)$ were resistant to MVB and all of them contained $b l a_{\mathrm{NDM}-1}$ or $b l a_{\mathrm{NDM}-5}$. The MICs of the remaining 52 strains $(52 / 58,89.66 \%)$ were in the range of $\leq 0.03125 / 8 \mu \mathrm{g} / \mathrm{mL}$ to $8 / 8 \mu \mathrm{g} / \mathrm{mL}$, which decreased by $2-128$ folds compared with meropenem alone. A total of 14 strains (14/58, 24.1\%) carrying $b l a_{\mathrm{KPC}-2}$ were highly susceptible to MVB, and their MICs decreased by 4-128 folds. Among the 14 strains, the MICs of 7 strains $(7 / 14,50 \%)$ were $\leq 0.03125 / 8 \mu \mathrm{g} / \mathrm{mL}$. The $\mathrm{MIC}_{50}$ and $\mathrm{MIC}_{90}$ of the strains carrying $b l a_{\mathrm{KPC}-2}$ decreased by 128 -fold and 16-fold, respectively. 
Table I Minimum Inhibitory Concentrations (MICs) of 58 Carbapenem-Resistant Escherichia coli Isolates

\begin{tabular}{|c|c|c|c|c|c|c|}
\hline \multirow[t]{2}{*}{ Isolates } & \multirow{2}{*}{$\begin{array}{c}\text { Fold } \\
\text { Change }\end{array}$} & \multicolumn{5}{|c|}{ MIC ( $\mu \mathrm{g} / \mathrm{mL})$} \\
\hline & & MEM & MVB & CAZIAVI & COL & TGC \\
\hline DC-38 & 4 & 0.25 & $0.0625 / 8$ & $64 / 4$ & 0.25 & 0.125 \\
\hline DC-269 & 2 & 2 & $1 / 8$ & $64 / 4$ & 0.125 & 0.25 \\
\hline DC-1918 & 2 & 0.25 & $0.125 / 8$ & $4 / 4$ & 0.125 & 0.125 \\
\hline DC- 1960 & 2 & 2 & $1 / 8$ & $\mathrm{I} / 4$ & 0.125 & 0.25 \\
\hline DC-2003 & 1 & 0.5 & $0.5 / 8$ & $64 / 4$ & 0.25 & 0.25 \\
\hline DC-3285 & 64 & 4 & $0.0625 / 8$ & $0.5 / 4$ & 0.5 & 0.25 \\
\hline DC-3737 & 1 & 16 & $16 / 8$ & $>256 / 4$ & 16 & 0.25 \\
\hline DC-3835 & 128 & 16 & $0.125 / 8$ & $1 / 4$ & 0.125 & 0.25 \\
\hline DC-3938 & 8 & 2 & $0.25 / 8$ & $0.5 / 4$ & 0.125 & 0.125 \\
\hline DC-4069 & 16 & 16 & $1 / 8$ & $8 / 4$ & 0.25 & 0.25 \\
\hline DC-4385 & 16 & 2 & $0.125 / 8$ & $8 / 4$ & 0.125 & 0.25 \\
\hline DC-4852 & 8 & I & $0.125 / 8$ & $0.5 / 4$ & 0.125 & 0.25 \\
\hline DC-4967 & 64 & 2 & $\leq 0.03 \mid 25 / 8$ & $<0.12 / 4$ & 0.25 & 0.25 \\
\hline DC-5108 & 64 & 4 & $\leq 0.03125 / 8$ & $0.25 / 4$ & 0.125 & 0.25 \\
\hline DC-5113 & 64 & 16 & $\leq 0.03125 / 8$ & $1 / 4$ & 0.5 & 0.25 \\
\hline DC-5II4 & 64 & 4 & $\leq 0.03125 / 8$ & $<0.12 / 4$ & 0.125 & 0.25 \\
\hline DC-5I27 & 8 & 0.125 & $0.0156 / 8$ & $<0.12 / 4$ & 0.25 & 0.25 \\
\hline DC-5I28 & I & 16 & $16 / 8$ & $<0.12 / 4$ & 0.5 & 0.25 \\
\hline DC-5I47 & 64 & 1 & $0.0156 / 8$ & $0.5 / 4$ & 0.25 & 0.25 \\
\hline DC-5I78 & 2 & 4 & $2 / 8$ & $0.5 / 4$ & 0.25 & 0.125 \\
\hline DC-5183 & 4 & 4 & $1 / 8$ & $0.25 / 4$ & 0.25 & 0.25 \\
\hline DC-5208 & 4 & 4 & $1 / 8$ & $1 / 4$ & 0.25 & $<0.0625$ \\
\hline DC-6525 & 2 & 0.125 & $0.0625 / 8$ & $<0.12 / 4$ & 0.25 & 0.125 \\
\hline DC-658I & 2 & 8 & $4 / 8$ & $\mathrm{I} / 4$ & 0.125 & 0.125 \\
\hline DC-6669 & 2 & 0.5 & $0.25 / 8$ & $1 / 4$ & 0.25 & 0.25 \\
\hline DC-6729 & 1 & 0.5 & $0.5 / 8$ & $>256 / 4$ & 0.25 & 0.125 \\
\hline DC-6824 & 16 & 2 & $0.125 / 8$ & $8 / 4$ & 0.25 & 0.25 \\
\hline DC-6834 & 8 & 0.25 & $0.03125 / 8$ & $1 / 4$ & 0.25 & 0.125 \\
\hline DC-6856 & 64 & 2 & $\leq 0.03125 / 8$ & $0.25 / 4$ & 0.25 & 0.25 \\
\hline DC-6896 & 64 & 2 & $\leq 0.03 / 25 / 8$ & $<0.12 / 4$ & 0.25 & 0.5 \\
\hline DC-6899 & 2 & 4 & $2 / 8$ & $0.25 / 4$ & 0.25 & $<0.0625$ \\
\hline DC-691I & I & I & $1 / 8$ & $>256 / 4$ & 0.25 & $<0.0625$ \\
\hline DC-7II4 & I & 4 & $4 / 8$ & $>256 / 4$ & 0.25 & $<0.0625$ \\
\hline DC-7|43 & I & 4 & $4 / 8$ & $>256 / 4$ & 0.25 & 0.125 \\
\hline DC-7I57 & 1 & 4 & $4 / 8$ & $8 / 4$ & 0.25 & $<0.0625$ \\
\hline DC-7333 & I & 2 & $2 / 8$ & $>256 / 4$ & 16 & $<0.0625$ \\
\hline DC-7350 & I & 8 & $8 / 8$ & $>256 / 4$ & 0.25 & $<0.0625$ \\
\hline DC-7368 & 1 & 2 & $2 / 8$ & $>256 / 4$ & 0.25 & 0.5 \\
\hline DC-7523 & 1 & 4 & $4 / 8$ & $>256 / 4$ & 0.5 & 0.5 \\
\hline DC-7603 & 2 & 16 & $8 / 8$ & $8 / 4$ & 0.5 & 0.5 \\
\hline DC-7658 & I & 8 & $8 / 8$ & $>256 / 4$ & 0.5 & 0.25 \\
\hline DC-7663 & I & 2 & $2 / 8$ & $8 / 4$ & 0.125 & 2 \\
\hline DC-7683 & I & 2 & $2 / 8$ & $8 / 4$ & 0.25 & 1 \\
\hline DC-7706 & I & 4 & $4 / 8$ & $>256 / 4$ & 0.5 & 0.5 \\
\hline DC-774I & I & 8 & $8 / 8$ & $>256 / 4$ & 0.5 & 2 \\
\hline DC-778I & I & 4 & $4 / 8$ & $>256 / 4$ & 0.25 & 0.25 \\
\hline DC-7782 & I & 16 & $16 / 8$ & $>256 / 4$ & 0.25 & 0.125 \\
\hline DC-7828 & 1 & 2 & $2 / 8$ & $0.25 / 4$ & 0.25 & I \\
\hline DC-79II & I & 16 & $16 / 8$ & $>256 / 4$ & 0.25 & I \\
\hline
\end{tabular}

(Continued) 
Table I (Continued).

\begin{tabular}{|c|c|c|c|c|c|c|}
\hline \multirow[t]{2}{*}{ Isolates } & \multirow{2}{*}{$\begin{array}{c}\text { Fold } \\
\text { Change }\end{array}$} & \multicolumn{5}{|c|}{ MIC $(\mu \mathrm{g} / \mathrm{mL})$} \\
\hline & & MEM & MVB & CAZIAVI & COL & TGC \\
\hline DC-79/4 & I & 16 & $16 / 8$ & $>256 / 4$ & 0.25 & I \\
\hline DC-7956 & 1 & 16 & $16 / 8$ & $>256 / 4$ & 0.5 & 2 \\
\hline DC-7969 & I & 0.125 & $0.125 / 8$ & $32 / 4$ & 0.125 & 0.125 \\
\hline DC-7980 & 1 & 0.5 & $0.5 / 8$ & $1 / 4$ & 0.25 & 1 \\
\hline DC-7994 & 64 & 1 & $0.0156 / 8$ & $0.25 / 4$ & 0.25 & 0.5 \\
\hline DC-8085 & I & 2 & $2 / 8$ & $64 / 4$ & 0.25 & 0.25 \\
\hline DC-8087 & I & 4 & $4 / 8$ & $8 / 4$ & 0.25 & 1 \\
\hline DC-8III & I & 4 & $4 / 8$ & $>256 / 4$ & 0.5 & 0.5 \\
\hline DC-8234 & 4 & 2 & $0.5 / 8$ & $1 / 4$ & 0.25 & 0.25 \\
\hline
\end{tabular}

Notes: Fold change, indicating the ratio of the MIC of Meropenem to the MIC of MVB. Bolded MICs values, indicating a strain resistant to corresponding drugs. Abbreviations: MEM, meropenem; MVB, meropenem-vaborbactam; CAZ/AVI, ceftazidime/avibactam; COL, colistin; TGC, tigecycline.

However, the MICs of 27 strains (27/58, 46.5\%) carrying $b l a_{\mathrm{NDM}}$ (except 3 strains) had no obvious changes. The MICs of strains carrying Cefotaximase-Munich (CTXM-)-type beta/Betalactam genes and bla $a_{\text {TEM-1 }}$ gene decreased by $2-8$ folds, indicating a minor change. All data are shown in Table 1 and Figure 1.

\section{Comparison of Susceptibility Rates with Regard to Diverse Separation Sites}

There was no significant difference in the MVB susceptibility rate among strains isolated from diverse separation sites and was the highest for blood (87.5\%), followed by urine and pus $(83.3 \%)$, and drainage fluid (81.8\%). The meropenem susceptibility rate was lower than the MVB susceptibility rate and was the highest for drainage fluid (36.4\%), followed by pus $(33.3 \%)$, and blood (25\%). Vaborbactam had a greater effect on meropenem susceptibility. There was no significant difference in various drug susceptibility rates of strains isolated from diverse separation sites (Table 2).

\section{Comparison of Susceptibility Rates with Regard to Diverse Resistance Genes}

MVB susceptibility did not vary significantly with regard to resistance genes carried by the strains. The higher MVB susceptibility was observed in the case of KPC strains $(92.9 \%$ susceptibility) and strains carrying CTX-M ( $85.7 \%$ susceptibility). The MVB susceptibility rate of strains carrying resistance genes was higher than that of the meropenem susceptibility rate. Furthermore, CAZ/AVI susceptibility was significantly higher in KPC isolates compared with that in the isolates carrying $b l a_{\mathrm{NDM}}$ $(P<0.05)$. There were non-significant differences in susceptibility rates among strains carrying other genes and towards other drugs (Table 3 ).

\section{Comparison of Susceptibility Rates with Regard to Diverse Combinations of Resistance Mechanisms}

A total of 14 combinations of resistance mechanisms were found in the strains (Table 4), and the most common combination was "New Delhi Metallo-beta/Betalactam (NDM) + CTX $+\mathrm{TEM}+\mathrm{ompC} / \mathrm{F}$ ", followed by CTX + ompC/F, KPC + CTX, and NDM + CTX + TEM. The susceptibility rate of MVB was significantly different among those groups including KPC and was different in the strains carrying both KPC and $\mathrm{NDM}$ at the same time. However, the $\mathrm{MIC}_{50}$ in groups including NDM was similar and the susceptibility rate was increased because of the increase of MVB breakpoint. The remaining combinations of resistance mechanisms involved the beta/Betalactam resistance genes (CTX and TEM) and outer membrane porin genes (ompC and ompF), and most of these strains were susceptible meropenem and MVB, except one isolate that carried TEM and ompC/F.

\section{Comparison of Susceptibility Rates with Regard to Diverse STs}

The MVB susceptibility rate was significantly different among strains belonging to different STs, and was the highest in ST8 (100\% susceptibility rate), and was $85.2 \%$ in other STs. Vaborbactam had a considerable effect on the restoration of meropenem susceptibility because the susceptibility percentage of meropenem in each ST group was lower. Compared with other ST groups, the susceptibility percentage 


\section{bla $_{\mathrm{KPC}}$}

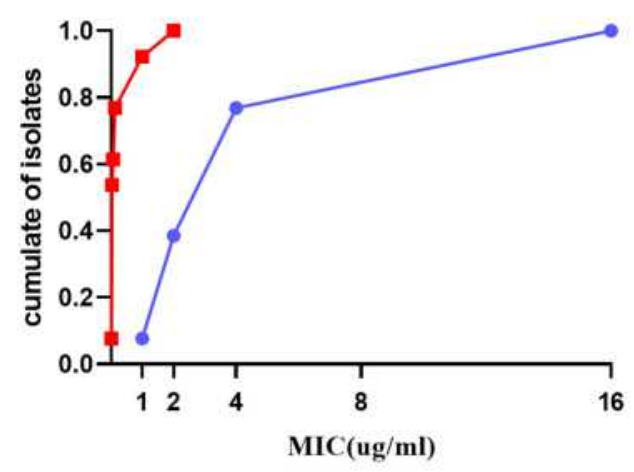

\section{bla $_{\text {TEM-1 }}$}
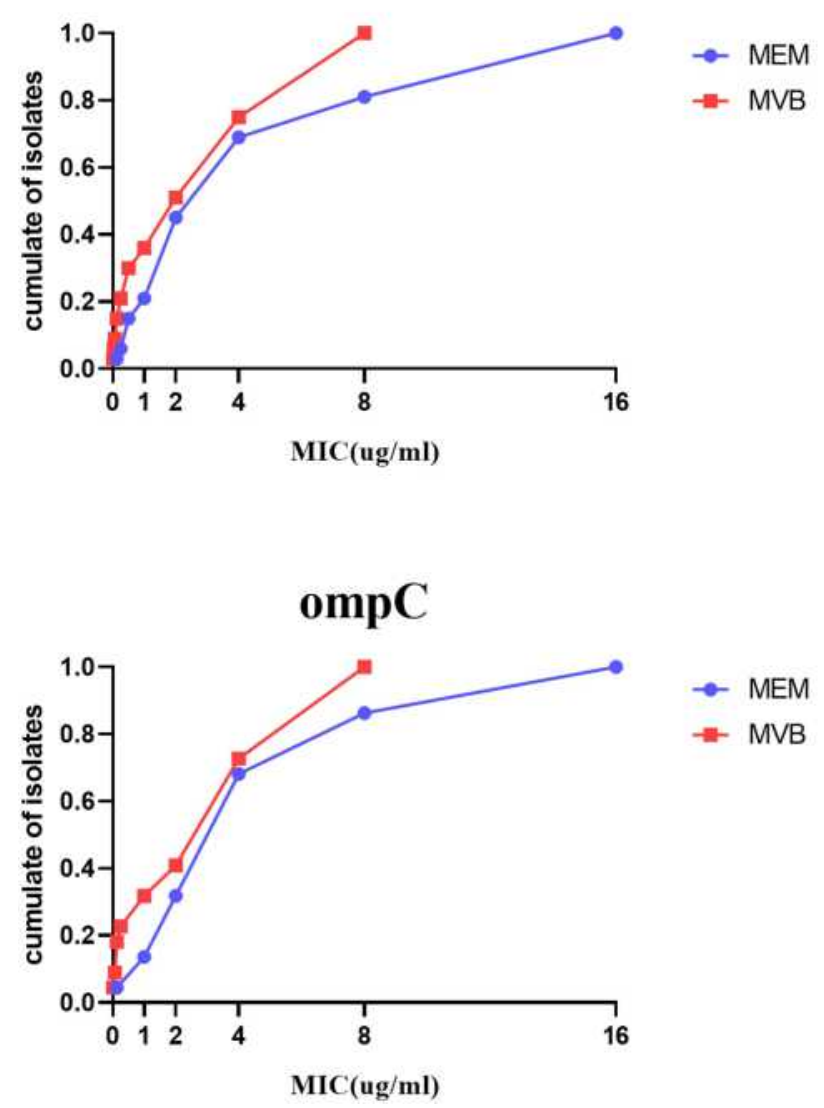

\section{bla $_{\mathrm{NDM}}$}

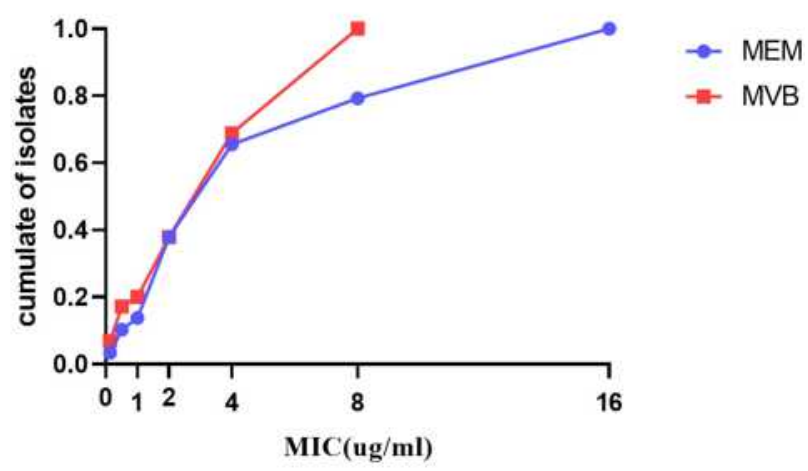

bla $_{\mathrm{CTX}-\mathrm{M}}$

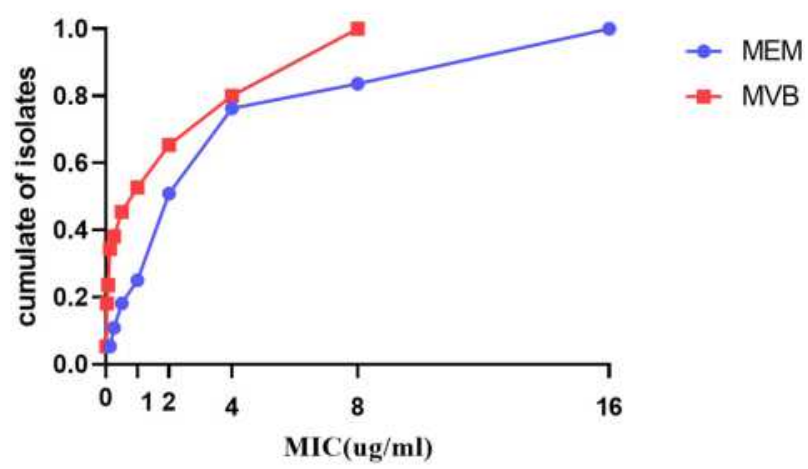

\section{ompF}

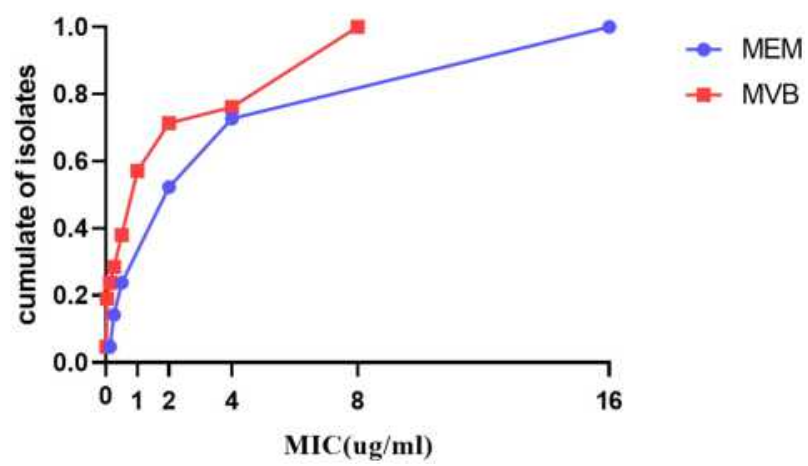

Figure I The cumulative percent of Escherichia coli isolates with different carbapenem-resistant mechanisms inhibited at specified meropenem-vaborbactam concentrations ( $\mu \mathrm{g} / \mathrm{mL}$ ).

of MVB, meropenem, and ceftazidime/avibactam in the ST8 group and the susceptibility percentage of amikacin in the ST19 group was statistically significant $(P<0.05)$ (Table 5).

\section{Discussion}

MVB, a new and effective anti-CREC drug, has expanded the spectrum of meropenem against CREC infection. However, its effects on different strains including CREC have not been evaluated. Therefore, this study aimed to evaluate the in vitro activity of MVB against CREC isolates and to compare the activity with other antibiotics with regard to different separation sites, carbapenemresistant mechanisms, and STs.

All 58 isolates showed higher resistance rates to commonly used drugs, including CAZ/AVI, which was an antibiotic with activity against serine-lactamase and had 
Table 2 Percent Susceptible ${ }^{\mathrm{a}}$ for Meropenem/Vaborbactam (MVB) and Comparator Agents in Relation to Different Separation Sites ${ }^{\mathrm{b}}$

\begin{tabular}{|c|c|c|c|c|c|c|}
\hline \multirow[t]{2}{*}{ Agent } & \multirow{2}{*}{$\begin{array}{c}\text { Total No. (\% of } \\
\text { 58) }\end{array}$} & \multicolumn{5}{|c|}{ Different Separation Sites, No, (Column \%) } \\
\hline & & $\begin{array}{l}\text { Urine } \\
(n=\mid 8)^{c}\end{array}$ & $\begin{array}{c}\text { Blood } \\
(n=16)^{c}\end{array}$ & $\begin{array}{l}\text { Drainage Fluid } \\
\qquad(n=I I)^{c}\end{array}$ & $\begin{array}{c}\text { Pus } \\
(n=6)^{c}\end{array}$ & $\begin{array}{l}\text { Other Separation Sites } \\
\qquad(\mathrm{n}=7)^{\mathrm{c}}\end{array}$ \\
\hline MVB & $48(82.8)$ & $15(83.3)$ & $14(87.5)$ & $9(81.8)$ & $5(83.3)$ & $5(7 \mid .4)$ \\
\hline MEM & $14(24.1)$ & $4(22.2)$ & $4(25.0)$ & $4(36.4)$ & $2(33.3)$ & 0 \\
\hline CAZ/AVI & $35(60.3)$ & II (6I.I) & $13(81.3)$ & $3(27.3)$ & $4(66.7)$ & $4(57.1)$ \\
\hline AMP & $\mathrm{I}(\mathrm{I} .7)$ & 0 & I (6.3) & 0 & 0 & 0 \\
\hline CRO & $\mathrm{I}(\mathrm{I} .7)$ & 0 & I (6.3) & 0 & 0 & 0 \\
\hline CAZ & $4(6.9)$ & 0 & I (6.3) & 0 & I (I6.7) & $2(28.6)$ \\
\hline IPM & $38(65.5)$ & $12(66.7)$ & $10(62.5)$ & $6(54.5)$ & $5(83.3)$ & 5 (7I.4) \\
\hline GEN & $8(13.8)$ & $4(22.2)$ & $2(12.5)$ & $2(18.2)$ & 0 & 0 \\
\hline TOB & $10(17.2)$ & $4(22.2)$ & $2(12.5)$ & $3(27.3)$ & 0 & I (14.3) \\
\hline AMK & $45(77.6)$ & $14(77.8)$ & $12(75.0)$ & $10(90.9)$ & $4(66.7)$ & 5 (7I.4) \\
\hline FOS & $36(62.1)$ & $13(8 \mid .3)$ & $12(75.0)$ & $5(45.5)$ & $4(66.7)$ & $2(28.6)$ \\
\hline
\end{tabular}

Notes: All isolates were susceptible to tigecycline. All isolates were non-susceptible to ertapenem, ciprofloxacin, levofloxacin and colistin. ${ }^{a}$ Data are expressed as no. isolates (column \%). ${ }^{b}$ Percent susceptible of isolates with separation sites irrespective. ${ }^{\mathrm{c}}$ There is no statistical significance $\left(P>0.05\right.$, according to $\chi^{2}$ test for two-group comparisons of all isolates).

Abbreviations: MVB, meropenem-vaborbactam; MEM, meropenem; CAZ/AVI, ceftazidime/avibactam; AMP, ampicillin; CRO, ceftriaxone; CAZ, ceftazidime; IPM, imipenem; ETP, ertapenem; CIP, ciprofloxacin; LVX, levofloxacin; GEN, gentamicin; TOB, tobramycin; AMK, amikacin; FOS, fosfomycin; COL, colistin.

Table 3 Percent Susceptible ${ }^{a}$ for Meropenem/Vaborbactam (MVB) and Comparator Agents in Relation to Individual Resistance Genes $^{b}$

\begin{tabular}{|c|c|c|c|c|c|c|c|}
\hline \multirow[t]{2}{*}{ Agent } & \multirow[t]{2}{*}{ Total No. (\% of 58 ) } & \multicolumn{2}{|c|}{$\begin{array}{c}\text { Carbapenem Resistance Genes, } \\
\text { No, (Column \%) }\end{array}$} & \multicolumn{2}{|c|}{$\begin{array}{c}\text { Beta/Betalactam Resistance } \\
\text { Genes, No, (Column \%) }\end{array}$} & \multicolumn{2}{|c|}{$\begin{array}{l}\text { Outer Membrane Porin } \\
\text { Genes, No, (Column \%) }\end{array}$} \\
\hline & & $\begin{array}{c}b^{b l a} a_{K P C} \\
(n=\mid 4)^{c}\end{array}$ & $\begin{array}{l}\text { bla }_{\text {NDM }} \\
(n=30)^{c}\end{array}$ & $\begin{array}{l}\text { bla } a_{C T X-M-X} \\
(n=56)^{c}\end{array}$ & $\begin{array}{l}b_{\text {TEM-1 }} \\
(n=34)^{c}\end{array}$ & $\begin{array}{c}\text { ompC } \\
(n=22)^{c}\end{array}$ & $\begin{array}{l}\text { ompF } \\
(n=21)^{c}\end{array}$ \\
\hline MVB & $48(82.8)$ & $13(92.9)$ & $21(70.0)$ & $48(85.7)$ & $26(76.5)$ & $16(72.7)$ & $16(76.2)$ \\
\hline MEM & $14(24.1)$ & I (7.I) & $4(13.3)$ & $14(25.0)$ & $7(20.6)$ & $3(13.6)$ & $5(23.8)$ \\
\hline CAZ/AVI & $35(60.3)$ & $12(85.7)^{*}$ & $10(33.3)^{*}$ & $34(60.7)$ & $14(4 \mid .2)$ & $12(54.5)$ & $15(71.4)$ \\
\hline AMP & I (I.7) & 0 & I (3.3) & I (I.8) & 0 & I (4.5) & 0 \\
\hline CRO & I (I.7) & 0 & $\mathrm{I}(3.3)$ & $\mathrm{I}(\mathrm{l} .8)$ & 0 & $\mathrm{I}(4.5)$ & 0 \\
\hline CAZ & $4(6.9)$ & 0 & I (3.3) & $4(7.1)$ & $2(5.9)$ & $4(18.2)$ & 0 \\
\hline IPM & $38(65.5)$ & $8(57.1)$ & $18(60.0)$ & $38(67.9)$ & $24(70.6)$ & $15(68.2)$ & $13(61.9)$ \\
\hline GEN & $8(13.8)$ & $2(14.3)$ & $4(13.3)$ & $7(12.5)$ & $3(8.8)$ & $3(13.6)$ & $2(9.5)$ \\
\hline TOB & $10(17.2)$ & $3(2 \mid .4)$ & $4(13.3)$ & $9(16.1)$ & $3(8.8)$ & $3(13.6)$ & $2(9.5)$ \\
\hline AMK & $45(77.6)$ & $12(85.7)$ & $20(66.7)$ & $43(76.8)$ & $26(75.0)$ & $17(77.3)$ & $14(66.7)$ \\
\hline FOS & $36(62.1)$ & $10(7 \mid .4)$ & $13(43.3)$ & $35(62.5)$ & $21(61.8)$ & $14(63.6)$ & $13(61.9)$ \\
\hline
\end{tabular}

Notes: All isolates were susceptible to tigecycline. All isolates were non-susceptible to ertapenem, ciprofloxacin, levofloxacin and colistin. All isolates did not carry bla $(\mathrm{n}=0)$, bla $a_{\mathrm{VIM}}(\mathrm{n}=0)$, bla $a_{\mathrm{OXA}-48}(\mathrm{n}=0)$ and bla $a_{\mathrm{SHV}}(\mathrm{n}=0)$. ${ }^{\mathrm{a}} \mathrm{Data}$ are expressed as no. isolates (column \%). ${ }^{\mathrm{b}}$ Percent susceptible of isolates with individual resistance genes irrespective of the presence/absence of other individual resistance genes. ${ }^{\mathrm{c}}$ Significant differences $\left(P<0.05\right.$, according to $\chi^{2}$ test for two-group comparisons of all isolates that have the indicated resistance gene) are in boldface and marked with an asterisk.

Abbreviations: MVB, meropenem-vaborbactam; MEM, meropenem; CAZ/AVI, ceftazidime/avibactam; AMP, ampicillin; CRO, ceftriaxone; CAZ, ceftazidime; IPM, imipenem; ETP, ertapenem; CIP, ciprofloxacin; LVX, levofloxacin; GEN, gentamicin; TOB, tobramycin; AMK, amikacin; FOS, fosfomycin; COL, colistin.

broad-spectrum activity against CREC isolates formerly, compared with those of colistin, tigecycline, and MVB. Similar results were reported by Brian D. Johnston et al; however, the resistance rate of amikacin was higher than that of our study $(26 \%$ vs. $19 \%) .{ }^{15}$ Therefore, the susceptibility results of MVB in CREC should be studied with a proper clinical niche. Among 58 CREC strains, the MICs of MVB for some strains decreased by 2-128 folds after the addition of vaborbactam. The considerably high in vitro activity was shown against some strains. The results of this study and those reported by Castanheira et $\mathrm{al}^{14}$ were similar, as both showed the potential MVB activity against contemporary CRE, CREC, and multi-drug resistance $E$. coli isolates. 
Table 4 Combination of Resistance Mechanisms Among 58 Carbapenem-Resistant Escherichia coli Isolates

\begin{tabular}{|c|c|c|c|c|c|c|c|}
\hline \multirow{2}{*}{$\begin{array}{l}\text { Combination of Resistance } \\
\text { Mechanisms }^{\text {a }}\end{array}$} & \multirow{2}{*}{$\begin{array}{l}\text { Total No. } \\
\text { (\% of 58) }\end{array}$} & \multicolumn{3}{|c|}{ Meropenem } & \multicolumn{3}{|c|}{ Meropenem/Vaborbactam } \\
\hline & & $\begin{array}{c}\mathrm{MIC}_{50} \\
(\mu \mathrm{g} / \mathrm{mL})\end{array}$ & $\begin{array}{c}\text { MIC Range } \\
(\mu \mathrm{g} / \mathrm{mL})\end{array}$ & $\begin{array}{c}\text { No. (\%) } \\
\text { Susceptible }\end{array}$ & $\begin{array}{c}\mathrm{MIC}_{50} \\
(\mu \mathrm{g} / \mathrm{mL})\end{array}$ & $\begin{array}{l}\text { MIC Range } \\
(\mu \mathrm{g} / \mathrm{mL})\end{array}$ & $\begin{array}{c}\text { No. (\%) } \\
\text { Susceptible }\end{array}$ \\
\hline $\mathrm{KPC}+\mathrm{CTX}$ & $8(13.8)$ & 4 & 1,16 & $\mathrm{I}(12.5)$ & $\leq 0.03125 / 8$ & $\leq 0.03125 / 8,1 / 8$ & $8(100)$ \\
\hline $\mathrm{KPC}+\mathrm{CTX}+\mathrm{TEM}$ & $\mathrm{I}(\mathrm{l} .7)$ & 16 & $-b$ & $0(0)$ & $2 / 8$ & $-{ }^{b}$ & $I(100)$ \\
\hline $\mathrm{KPC}+\mathrm{CTX}+\mathrm{ompC} / \mathrm{F}$ & $3(1.7)$ & 2 & 2,4 & $0(0)$ & $\leq 0.03125 / 8$ & $\leq 0.03125 / 8,1 / 8$ & $3(100)$ \\
\hline $\mathrm{KPC}+\mathrm{CTX}+\mathrm{TEM}+\mathrm{ompC} / \mathrm{F}$ & $\mathrm{I}(\mathrm{I} .7)$ & 4 & $-b$ & $0(0)$ & $2 / 8$ & - b & $I(100)$ \\
\hline $\mathrm{KPC}+\mathrm{NDM}+\mathrm{CTX}+\mathrm{TEM}+\mathrm{ompC} / \mathrm{F}$ & $\mathrm{I}(\mathrm{I} .7)$ & 2 & $-b$ & $0(0)$ & $0.5 / 8$ & $-b$ & $I(100)$ \\
\hline $\mathrm{NDM}+\mathrm{CTX}$ & $\mathrm{I}(\mathrm{I} .7)$ & 4 & $-b$ & $0(0)$ & $4 / 8$ & $-\mathrm{b}$ & $I(100)$ \\
\hline $\mathrm{NDM}+\mathrm{CTX}+\mathrm{ompC} / \mathrm{F}$ & $3(5.2)$ & 16 & 2,16 & $0(0)$ & $16 / 8$ & $2 / 8,16 / 8$ & I (33.3) \\
\hline $\mathrm{NDM}+\mathrm{TEM}+\mathrm{ompC} / \mathrm{F}$ & $2(3.4)$ & 4 & 4,8 & $0(0)$ & $4 / 8$ & $4 / 8,8 / 8$ & I (50) \\
\hline $\mathrm{NDM}+\mathrm{CTX}+\mathrm{TEM}$ & $8(13.8)$ & 1 & $0.125,2$ & $4(50)$ & $1 / 8$ & $0.125 / 8,2 / 8$ & $8(100)$ \\
\hline $\mathrm{NDM}+\mathrm{CTX}+\mathrm{TEM}+\mathrm{ompC} / \mathrm{F}$ & $15(25.9)$ & 4 & 2,16 & $0(0)$ & 4 & $0.125 / 8,16 / 8$ & $9(60)$ \\
\hline CTX+TEM & I (I.7) & 0.25 & $-b$ & $I(100)$ & $0.0625 / 8$ & - b & $I(100)$ \\
\hline $\mathrm{CTX}+o \mathrm{mp} \mathrm{C} / \mathrm{F}$ & $9(15.5)$ & 0.5 & $0.125,4$ & $6(66.7)$ & $0.125 / 8$ & $0.03125 / 8,2 / 8$ & $9(100)$ \\
\hline$C T X+T E M+o m p C / F$ & $4(6.9)$ & 0.5 & $0.5,2$ & $2(50)$ & 0.25 & $0.0156 / 8,1 / 8$ & $4(100)$ \\
\hline $\mathrm{TEM}+\mathrm{ompC} / \mathrm{F}$ & $\mathrm{I}(\mathrm{I} .7)$ & 16 & $-b$ & $0(0)$ & $8 / 8$ & - b & $0(0)$ \\
\hline
\end{tabular}

Notes: ${ }^{\mathrm{a} C o m b i n a t i o n ~ o f ~ r e s i s t a n c e ~ m e c h a n i s m s ~ a r e ~ l i s t e d ~ a l p h a b e t i c a l l y ~(p r i o r i t i s i n g ~ c a r b a p e n e m a s e s) ; ~}{ }^{\mathrm{b}}$-, Indicating not applicable (only one isolate).

In fact, KPC-producing CRE infections are associated with a significantly higher risk of overall mortality compared with that of non-KPC-producing CRE infections. ${ }^{3}$ In our research, it is worth noting that MVB, CAZ/AVI, and AMK were active against KPC-producers, which was an Ambler class A enzyme that utilizes serine at the active

Table 5 Percent Susceptible ${ }^{\mathrm{a}}$ for Meropenem/Vaborbactam (MVB) and Comparator in Relation to Sequence Types (STs) ${ }^{\mathrm{b}}$

\begin{tabular}{|c|c|c|c|c|c|}
\hline \multirow[t]{2}{*}{ Agent } & \multirow{2}{*}{$\begin{array}{l}\text { Total No. } \\
\text { (\% of } 58 \text { ) }\end{array}$} & \multicolumn{4}{|c|}{ ST, No, (Column \%) } \\
\hline & & $\begin{array}{c}\text { ST8 } \\
(n=17)^{c}\end{array}$ & $\begin{array}{l}\text { ST19 } \\
(n=7)^{c}\end{array}$ & $\begin{array}{l}\text { ST692 } \\
(n=7)^{c}\end{array}$ & $\begin{array}{c}\text { Other STs } \\
(n=27)^{c}\end{array}$ \\
\hline MVB & $48(82.8)$ & $17(100)^{*}$ & $4(57.1)$ & $4(57.1)$ & $23(85.2)$ \\
\hline MEM & $14(24.1)$ & $4(23.5)^{*}$ & 0 & I (14.3) & $9(33.3)$ \\
\hline CAZI & $35(60.3)$ & $15(88.2) *$ & $2(28.6)$ & I (14.3) & $17(63.0)$ \\
\hline $\mathrm{AVI}$ & & & & & \\
\hline AMP & $\mathrm{I}(\mathrm{I} .7)$ & 0 & 0 & 0 & I (3.7) \\
\hline CRO & I (I.7) & 0 & 0 & 0 & I (3.7) \\
\hline CAZ & $4(6.9)$ & I (5.9) & 0 & 0 & $3(11.1)$ \\
\hline IPM & $38(65.5)$ & $12(70.6)$ & $2(28.6)$ & $5(71.4)$ & $19(70.4)$ \\
\hline GEN & $8(13.8)$ & $4(23.5)$ & I (14.3) & 0 & $3(11.1)$ \\
\hline ТОВ & $10(17.2)$ & $5(29.4)$ & I (14.3) & 0 & $4(14.8)$ \\
\hline AMK & $45(77.6)$ & $16(94.1)$ & 2 (28.6)* & $5(71.4)$ & $22(81.5)$ \\
\hline FOS & $36(62.1)$ & $14(82.4)$ & $2(28.6)$ & $4(57.1)$ & $16(59.3)$ \\
\hline
\end{tabular}

Notes: All isolates were susceptible to tigecycline. All isolates were nonsusceptible to ertapenem, ciprofloxacin, levofloxacin and colistin. ${ }^{a}$ Data are

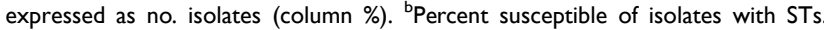
'Significant differences $\left(P<0.05\right.$, according to $\chi^{2}$ test for two-group comparisons of all isolates) are in boldface and marked with an asterisk.

Abbreviations: MVB, meropenem-vaborbactam; MEM, meropenem; CAZ/AVI, ceftazidime/avibactam; AMP, ampicillin; CRO, ceftriaxone; CAZ, ceftazidime; IPM, imipenem; ETP, ertapenem; CIP, ciprofloxacin; LVX, levofloxacin; GEN, gentamicin; TOB, tobramycin; AMK, amikacin; FOS, fosfomycin; COL, colistin. site to facilitate hydrolysis of nearly all currently available beta/Betalactam antibiotics, ${ }^{16}$ and $50 \%$ of KPCproducing CREC isolates were susceptible to MVB (MIC $\leq 0.003125 / 8 \mu \mathrm{g} / \mathrm{mL}$ ) and this was in line with Zhou et al. ${ }^{17} \mathrm{~A}$ previous study reported that a combination of MVB with various carbapenems reduced the MIC values by 16-64 folds for tested KPCproducing isolates in the US. ${ }^{18}$ Our finding expressed that MVB had the highest decrease in MIC values for KPCproducing CREC strains isolated from southeastern China. On the other hand, the MICs of strains carrying CTX-M-type beta/Betalactam genes, bla ${ }_{\mathrm{TEM}-1}$ genes, and outer membrane porin genes were different; however, the extent of decrease was less than that of KPC. Probably the inhibitory activity of vaborbactam is less important when it meets class A ESBLs and class C cephalosporinases alone ${ }^{19}$ compared with that of the stable carbapenem. In other words, the low off rate of vaborbactam for KPC plays an important role in enhancing the activities of antibiotics against KPC-producing strains, and the low potency of vaborbactam against CTX- and TEMproducing strains might be that it cannot form a stable inhibitory complex with these enzymes. ${ }^{19}$ However, our study showed that MVB and CAZ/AVI did not exhibit strong inhibitory activity against the Metallo-beta /Betalactam NDM-1 and NDM-5, and was consistent with the results reported by Lomovskaya et al. ${ }^{20}$ As vaborbactam and avibactam lack inhibitory activity against Metallo-beta/Betalactam, it resulted in a similar 
MIC distribution for MVB and meropenem, with the $\mathrm{MIC}_{50}$ and $\mathrm{MIC}_{90}$ of both the two drugs were 16 and $32 \mathrm{mg} / \mathrm{L}$, respectively. ${ }^{14,21}$ However, the $\mathrm{MIC}_{50}$ and $\mathrm{MIC}_{90}$ of $\mathrm{MVB}$ and meropenem of the NDM group were lower in our study (Table 1). The percent susceptibility of MVB was higher than that of meropenem in the NDM group (Tables 3 and 4). It was not only related to Metallo-beta/Betalactam, but also the increase of MVB breakpoint. This phenomenon was observed in 14 combinations of resistance mechanisms; MVB exhibited considerably high activity even when both KPC and NDM were present in the same strain.

MVB has been approved by the FDA for the treatment of complicated urinary tract infections. ${ }^{7}$ The CRCE strains isolated from various separation sites showed susceptibility towards MVB, and had similarities with no statistical differences, suggesting that MVB might be effective against CREC infections of various sources. This observation needs to be verified with more isolates and in vivo tests. Additionally, MVB activity varied significantly with regard to STs; the susceptibility of ST8 strains towards MVB, meropenem, and CAZ/ AVI had statistical differences. Notably, ST8 was the predominant ST in the present study; however, other studies have reported that ST131 H30R1 and H30Rx subclones are more susceptible to MVB than isolates of other STs. ${ }^{12,22}$

To summarize, MVB is effective against most CREC clinical isolates found in the hospital of southeastern China, and the susceptibility of CREC strains towards MVB varied with regard to carbapenem-resistant mechanisms and STs. MVB might be considered an alternative treatment against invasive infections caused by KPC-producing and ST8 CREC isolates. It requires the determination of the type of carbapenemase and susceptibility to MVB and/or STs.

\section{Ethical Statement}

The study was approved by the Ethics Committee of the First Affiliated Hospital of Wenzhou Medical University. This article did not conduct human or animal studies, so informed consent was abandoned.

\section{Acknowledgments}

This study was supported by the Planned Science and Technology Project of Wenzhou (no. Y20170204).

\section{Author Contributions}

All authors made a significant contribution to the work reported, whether that is in the conception, study design, execution, acquisition of data, analysis and interpretation, or in all these areas; took part in drafting, revising or critically reviewing the article; gave final approval of the version to be published; have agreed on the journal to which the article has been submitted; and agree to be accountable for all aspects of the work.

\section{Disclosure}

The authors report no conflicts of interest in this work.

\section{References}

1. Luepke K, Mohr J. The antibiotic pipeline: reviving research and development and speeding drugs to market. Expert Rev Anti Infect Ther. 2017;15(5):425-433. doi:10.1080/14787210.2017.1308251

2. Falagas M, Mavroudis A, Vardakas K. The antibiotic pipeline for multi-drug resistant gram negative bacteria: what can we expect? Expert Rev Anti Infect Ther. 2016;14(8):747-763. doi:10.1080/ 14787210.2016.1204911

3. Meng X, Liu S, Duan J, et al. Risk factors and medical costs for healthcare-associated carbapenem-resistant Escherichia coli infection among hospitalized patients in a Chinese teaching hospital. $B M C$ Infect Dis. 2017;17(1):82. doi:10.1186/s12879-016-2176-9

4. Schwaber M, Carmeli Y. Carbapenem-resistant Enterobacteriaceae: a potential threat. J Am Med Assoc. 2008;300(24):2911-2913.

5. Sheu C, Chang Y, Lin S, et al. EnterobacteriaceaeInfections caused by carbapenem-resistant: an update on therapeutic options. Front Microbiol. 2019;10:80. doi:10.3389/fmicb.2019.00080

6. Doi Y. Treatment options for carbapenem-resistant Gram-negative bacterial infections. Clin Infect Dis. 2019;69:S565-S575. doi:10.1093/cid/ciz830

7. Burgos R, Biagi M, Rodvold K, et al. Pharmacokinetic evaluation of meropenem and vaborbactam for the treatment of urinary tract infection. Expert Opin Drug Metab Toxicol. 2018;14 (10):1007-1021. doi:10.1080/17425255.2018.1511702

8. Gibson B, Brief A. Review of a new antibiotic: meropenem-vaborbactam. Sr Care Pharm. 2019;34(3):187-191.

9. Hackel M, Lomovskaya O, Dudley M, et al. In vitro activity of meropenem-vaborbactam against clinical isolates of KPC-positive Enterobacteriaceae. Antimicrob Agents Chemother. 2018;62(1). doi:10.1128/AAC.01904-17

10. Dafopoulou K, Xavier B, Zarkotou O, et al. Detection of colistin resistance in the highly virulent Escherichia coli ST131 H30Rx clone in Greece. J Glob Antimicrob Resist. 2020;20:31-32. doi:10.1016/j. jgar.2019.11.014

11. Johnson J, Johnston B, Kuskowski M, et al. Intensity and mechanisms of fluoroquinolone resistance within the $\mathrm{H} 30$ and H30Rx subclones of Escherichia coli sequence type 131 compared with other fluoroquinolone-resistant E. coli. Antimicrob Agents Chemother. 2015;59(8):4471-4480. doi:10.1128/AAC.00673-15

12. Johnson J, Porter S, Thuras P, et al. Greater ciprofloxacin tolerance as a possible selectable phenotype underlying the pandemic spread of the H30 subclone of Escherichia coli sequence type 131. Antimicrob Agents Chemother. 2015;59(11):7132-7135. doi:10.1128/AAC.01687-15

13. Tian X, Zheng X, Sun Y, et al. Escherichia molecular mechanisms and epidemiology of carbapenem-resistant isolated from Chinese patients during 2002-2017. Infect Drug Resist. 2020;13:501-512. doi:10.2147/IDR.S232010 
14. Castanheira M, Huband M, Mendes R, et al. Meropenemvaborbactam tested against contemporary Gram-negative isolates collected Worldwide during 2014, including carbapenem-resistant, KPC-producing, multidrug-resistant, and extensively drug-resistant Enterobacteriaceae. Antimicrob Agents Chemother. 2017;61(9). doi:10.1128/AAC.00567-17

15. Johnston B, Thuras P, Porter S, et al. Activity of meropenem/vaborbactam against international carbapenem-resistant Escherichia coli isolates in relation to clonal background, resistance genes, resistance to comparators and region. $J$ Glob Antimicrob Resist. 2021;24:190-197. doi:10.1016/j.jgar.2020.12.017

16. Jorgensen S, Rybak M. Meropenem and vaborbactam: stepping up the battle against carbapenem-resistant Enterobacteriaceae. Pharmacotherapy. 2018;38(4):444-461. doi:10.1002/phar.2092

17. Zhou M, Yang Q, Lomovskaya O, et al. In vitro activity of meropenem combined with vaborbactam against KPC-producing Enterobacteriaceae in China. J Antimicrob Chemother. 2018;73 (10):2789-2796. doi:10.1093/jac/dky251

18. Hecker S, Reddy K, Totrov M, et al. Discovery of a cyclic boronic acid $\beta$-lactamase inhibitor (RPX7009) with utility vs class A serine carbapenemases. J Med Chem. 2015;58(9):3682-3692. doi:10.1021/ acs.jmedchem.5b00127
19. Wozniak A, Villagra N, Undabarrena A, et al. Porin alterations present in non-carbapenemase-producing Enterobacteriaceae with high and intermediate levels of carbapenem resistance in Chile. J Med Microbiol. 2012;61:1270-1279. doi:10.1099/jmm.0.045799-0

20. Lomovskaya O, Sun D, Rubio-Aparicio D, et al. Vaborbactam: spectrum of beta-lactamase inhibition and impact of resistance mechanisms on activity in Enterobacteriaceae. Antimicrob Agents Chemother. 2017;61(11). doi:10.1128/AAC.01443-17

21. Pfaller M, Huband M, Mendes R, et al. In vitro activity of meropenem/vaborbactam and characterisation of carbapenem resistance mechanisms among carbapenem-resistant Enterobacteriaceae from the 2015 meropenem/vaborbactam surveillance programme. Int $J$ Antimicrob Agents. 2018;52(2):144-150. doi:10.1016/j. ijantimicag.2018.02.021

22. Johnson J, Porter S, Thuras P, et al. Epidemic emergence in the United States of Escherichia coli sequence type 131-30 (ST131-30), 2000 to 2009. Antimicrob Agents Chemother. 2017;61(8). doi:10.1128/AAC.00732-17
Infection and Drug Resistance

\section{Publish your work in this journal}

Infection and Drug Resistance is an international, peer-reviewed openaccess journal that focuses on the optimal treatment of infection (bacterial, fungal and viral) and the development and institution of preventive strategies to minimize the development and spread of resistance. The journal is specifically concerned with the epidemiology of
Dovepress

antibiotic resistance and the mechanisms of resistance development and diffusion in both hospitals and the community. The manuscript management system is completely online and includes a very quick and fair peerreview system, which is all easy to use. Visit http://www.dovepress.com/ testimonials.php to read real quotes from published authors. 